\title{
MUTI-COLOR IMAGING OF CLUSTERS OF GALAXIES WITH MOSAIC CCD CAMERAS
}

\author{
S. OKAMURA ${ }^{1}$, M. DOI $^{1}$, N. KASHIKAWA ${ }^{2}$, W. KAWASAKI ${ }^{1}$, \\ Y. KOMIYAMA ${ }^{1}$, M. SEKIGUCHI ${ }^{2}$, K. SHIMASAKU ${ }^{1}$, \\ M. YAGI ${ }^{1}$ AND N. YASUDA ${ }^{1}$ \\ ${ }^{1}$ Department of Astronomy and Research Center for the Early \\ Universe, University of Tokyo, Japan \\ ${ }^{2}$ National Astronomical Obvservatory of Japan
}

\section{Introduction}

At present, the photometric data for clusters at $\mathrm{z} \lesssim 0.2$ mainly come from photographic photometry. The lack of CCD data for such clusters is simply due to the fact that no CCD camera had been available until recently that covers the wide extension of clusters within a reasonable amount of observing time. We have developed a large mosaic CCD camera and conducted multicolor imaging observations of $z \lesssim 0.2$ clusters using the 40 -inch Swope telescope at Las Campanas Observatory.

\section{2. $5 \mathrm{~K} \times 8 \mathrm{~K}$ Mosaic CCD Camera}

Our mosaic CCD camera consists of forty $1 \mathrm{~K} \times 1 \mathrm{~K}$ CCDs located on a grid of the $8 \times 5$ array. The CCDs are TC-215 with 12 -micron pixels manufactured by Texas Instruments Japan. The spacing between adjacent CCDs is slightly smaller than twice the side of the CCD. The image of a contiguous field, which consists of $14,500 \times 9,280$ pixels, is composed of four offset exposures. Figure 1 shows the camera as of April 1996. A brief description of the camera is given by Kashikawa et al. (1995a)

\section{Observations and Data Reductions}

We observed more than a dozen nearby $(z \lesssim 0.2)$ clusters of galaxies in the $B, V$, and $R$ bands in 1994-5. The camera evolved during this period from the $4 \times 7$ array to the $5 \times 8$. At the Cassegrain focus of the Swope telescope, 


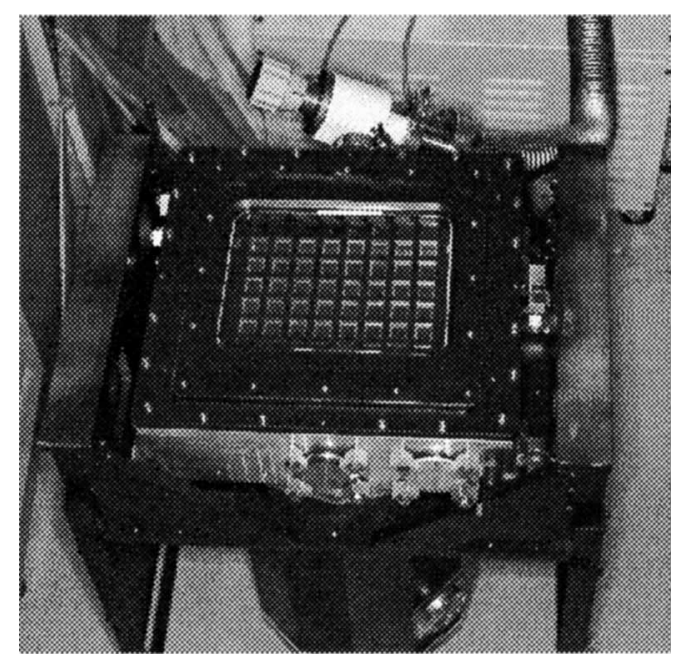

Figure 1. The $5 \mathrm{~K} \times 8 \mathrm{~K}$ mosaic $\mathrm{CCD}$ camera

the camera $(5 \times 8$ array) gave a field of view of about $1.41 \times 0.90=1.27$ square degrees with the resolution of 0.35 arcsec pixel ${ }^{-1}$.

The data are processed with a software package dedicated to our camera. In addition to the pre-processings common to CCD data such as bias/dark subtraction and flat fielding, we need to do the frame mosaicking and PSF equalization, which are pre-processings characteristic of our camera. The former is to establish a consistent coordinate system and a consistent flux scale while the latter is to assure the uniformity of object detection and extracted parameters, over a contiguous field composed of four separate offset exposures taken by 40 different chips. The mosaicked and PSFequalized data of a contiguous field is subjected to the more or less usual image analysis consisting of object detection, measurement of photometric and shape parameters, and star/galaxy discrimination.

\section{Results}

Based on the $R$-band data of four clusters, A1656 (Coma; $z=0.02$ ), A1367 $(z=0.02)$, A1644 $(z=0.05)$, and A1631 $(z=0.05)$, we detected a significant cluster-to-cluster variation in the faint part $\left(M_{R} \gtrsim-18 \mathrm{mag}\right)$ of the luminosity function of early-type galaxies (Kashikawa et al. 1995b). The data for A1656 and A1367 were obtained with our old mosaic CCD camera attached to the Kiso Schmidt telescope (Sekiguchi et al. 1992).

Our morphological classification (Doi et al. 1993) is based solely on the shape of the luminosity distribution in a single color band ( $R$ band), which is subject to the seeing effect. In order to examine the reliability of our 


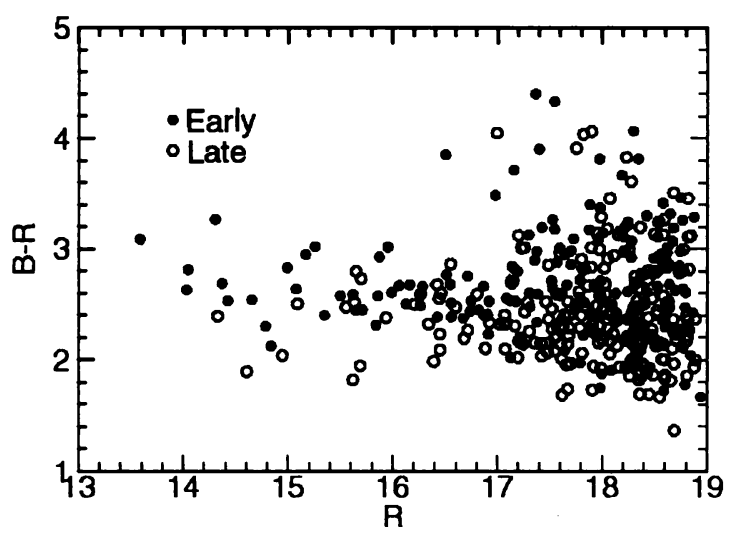

Figure 2. $\quad B-R$ colors (zero point arbitrary) of galaxies in A1631 as a function of $R$ magnitude

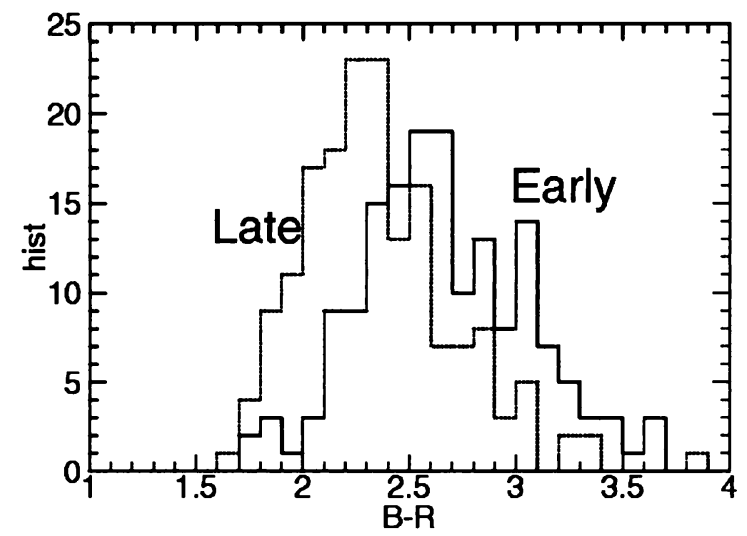

Figure 3. $\quad B-R$ colors (zero point arbitrary) of galaxies in A1631 as a function of $B-R$ colors

classification we analyzed the $B$-band data of A1631 and obtained the $B-R$ color for galaxies. The colors are computed from the aperture magnitudes. Aperture radius is set to be $1.5 r_{\mathrm{e}}$, where $r_{\mathrm{e}}$ is the isophotal equivalent radius measured in the $R$ band, i.e., $r_{\mathrm{e}}=\{(\text { isophotal image area }) / \pi\}^{1 / 2}$. The internal photometric accuracy is estimated as $\lesssim 0.08 \mathrm{mag}$ in r.m.s. in $m_{R}<18.5$.

Figure 2 shows the $B-R$ relative color as a function of $R$ magnitude while Figure 3 shows the histograms for the two types. The histogram of 
the early-type is shifted to the red with respect to that of the late-type by about $0.3 \mathrm{mag}$. This systematic difference is taken to be evidence that our morphological classification is broadly valid at $z \sim 0.05$.

\section{References}

Doi, M., Fukugita, M., \& Okamura, S. 1993, Mon.Not.R.astron.Soc., 264, 832.

Kashikawa, N. et al. 1995a, in "Scientific and Engineering Frontiers for 8-10m Telescopes," eds. M.Iye and T.Nishimura(Tokyo: Universal Academy Press), pp.105-110.

Kashikawa, N. et al. 1995b, Astrophys.J., 452, L99.

Sekiguchi, M. et al. 1992, Publ.Astron.Soc.Pacific, 104, 744. 